\title{
The management of contaminated goods in Japan since the Fukushima accident
}

\author{
M. Maître ${ }^{1, *, a}$, P. Croüail ${ }^{1}$, V. Durand ${ }^{2}$, J.-F. Lecomte ${ }^{2}$, S. Charron ${ }^{2}$ and T. Schneider ${ }^{1}$ \\ ${ }^{1}$ Nuclear Protection Evaluation Center (CEPN), Fontenay-aux-Roses, France. \\ ${ }^{2}$ Radiation Protection and Nuclear Safety Institute (IRSN), Fontenay-aux-Roses Cedex, France.
}

Received: 7 January 2020 / Accepted: 30 January 2020

\begin{abstract}
Feedback analyses from Chernobyl and Fukushima post-accident situations have clearly emphasized the importance of management strategies for contaminated goods during the days, months and years after the accident. For instance, following the accident, the management of contaminated goods generally focuses on the implementation of protective actions, radiological countermeasures and the control of radioactivity in foodstuffs, in order to ensure the quality and sales of the food products. Then, issues concerning the loss of image of local products can appear, and subsequent strategies have to be taken to restore the consumer confidence. Based on a literature review and interviews performed between 2016 and 2018 with Japanese stakeholders involved in the Fukushima accident recovery, this paper analyses the strategies for the management of contaminated goods which have been implemented in Japan. Divided into three main parts, this paper highlights how producers/livestock breeders, agricultural cooperatives, consumers and even distributors gradually committed themselves to (i) ensure the radiological quality of products by implementing countermeasures and monitoring systems, (ii) maintain sustainable economic activity by encouraging the resumption of agricultural activities and by deploying various sales strategies and try to (iii) restore consumer confidence by initiating producer/consumer dialogues and promotional actions for local products.
\end{abstract}

Keywords: Fukushima / foodstuff / radiological monitoring / economic strategies / consumer confidence

\section{Introduction}

The accidents of Windscale (1957), Chernobyl (1986) and Fukushima (2011) have demonstrated the importance of implementing appropriate strategies for managing contaminated goods, especially those intended for human consumption. Indeed, while the first challenge following a nuclear accident is to protect the population from the immediate impacts of radioactive releases, it appears that in the long term, the effects of radioactive deposits in the environment are the main source of concern for the population and public authorities. These radioactive deposits can lead to either external exposure, through direct radiation, or internal contamination, mainly via the ingestion of contaminated food. In such a context, it is important to establish radiological monitoring procedures and appropriate protective measures in order to guarantee the radiological quality of products and thus ensure the long-term protection of the population. Moreover,

\footnotetext{
*Corresponding author: melanie.maitre@cepn.asso.fr

${ }^{a}$ Present address: 28, rue de la Redoute, 92260 Fontenay-aux-Roses, France.
}

feedback from Chernobyl and Fukushima disasters have highlighted other issues that go well beyond the management of exposure to radiation. For example, the harm to the reputation of products from regions affected by nuclear accidents, and the consequences for these regions' economies, pose a real challenge, in response to which producers, cooperatives, distributors and even consumers are required to develop innovative approaches.

Several years after the Fukushima accident, the aim of this article is to provide feedback analysis on the management of contaminated food in Japan. More specifically, it describes (i) the countermeasure and radiological monitoring strategies put in place by the Japanese authorities and by production sectors, (ii) the economic incentives developed to promote the revitalisation of agricultural activities, and finally (iii) the actions undertaken to try to restore consumer confidence.

\section{Methodology}

The analysis provided in this article is largely based on interviews carried out with various Japanese stakeholders involved in the management of contaminated goods, including 
farmers, livestock breeders, fishermen, agricultural cooperatives of producers, consumer associations and representatives of national, prefectoral and municipal authorities. Between 2016 and 2018, several visits to Japan were organised, in order to meet these various stakeholders and study the management responses of the most representative production sectors of the Fukushima Region: dairy farming, suckler farming, rice farming, fruit farming (e.g. peaches, persimmons, etc.) and sea fishing. The semi-structured interviews sought detailed understanding of the countermeasures and radiological monitoring operations carried out both by the Japanese authorities and at sectorial levels. Information on consumers' and distributors' perceptions and behaviours, both inside and outside Fukushima Prefecture, was also gathered. Furthermore, the interviews aimed to collect information on the sales strategies developed by producers and distributors to compensate for the loss of reputation of their local products. The analysis also considers testimonies shared in the context of the dialogue initiative undertaken with the inhabitants of Fukushima Prefecture by the International Commission on Radiological Protection (ICRP) since fall 2011 (Kotoba, 2015; Lochard et al., 2019). Alongside these elements and on the basis of the work carried out as part of the European PREPARE research project (Raskob, 2016), the review of articles dedicated to food management following the Fukushima accident was completed. Reports and results of studies dedicated to the management of contaminated goods and published by the Japanese authorities (e.g. Fukushima Prefecture, the Ministry of Agriculture, Forestry and Fisheries (MAFF), the Ministry of Health, Labour and Welfare (MHLW), etc.) were also consulted. Finally, results were shared and discussed over the course of the three years study with a group of European experts brought together in a working group dedicated to the management of contaminated goods in post-accident situations, namely the "ConGoo" (Contaminated Goods) working group of the European platform on preparedness for nuclear and radiological emergency response and recovery (NERIS - eu-neris.net).

\section{Feedback on the management of contaminated goods during the first weeks following the accident}

The Tōhoku Region covers all of northeastern Japan and is made up of six prefectures, including Fukushima. Mainly based on a rural economy, before 2011, this region was considered as the "rice granary, vegetable garden and orchard of the Greater Tokyo Region". The nuclear accident that occurred at the Fukushima-Daiichi nuclear power plant (NPP) on 11 March 2011 caused serious damage to the Tōhoku Region, which agricultural activities were particularly affected, leading to a food crisis that was felt throughout the whole country (Ishii and Morlans, 2013).

However, the weather conditions and the date of the Fukushima accident, which occurred at the end of winter 2011, limited the quantity and impact of radioactive deposits. As a matter of fact, the vast majority (more than $80 \%$ ) of the radioactive plume dispersed towards the Pacific Ocean, limiting the amount of radioactive deposits on land (Ohara and Miyahara, 2016). Moreover, at this time of year (i.e.

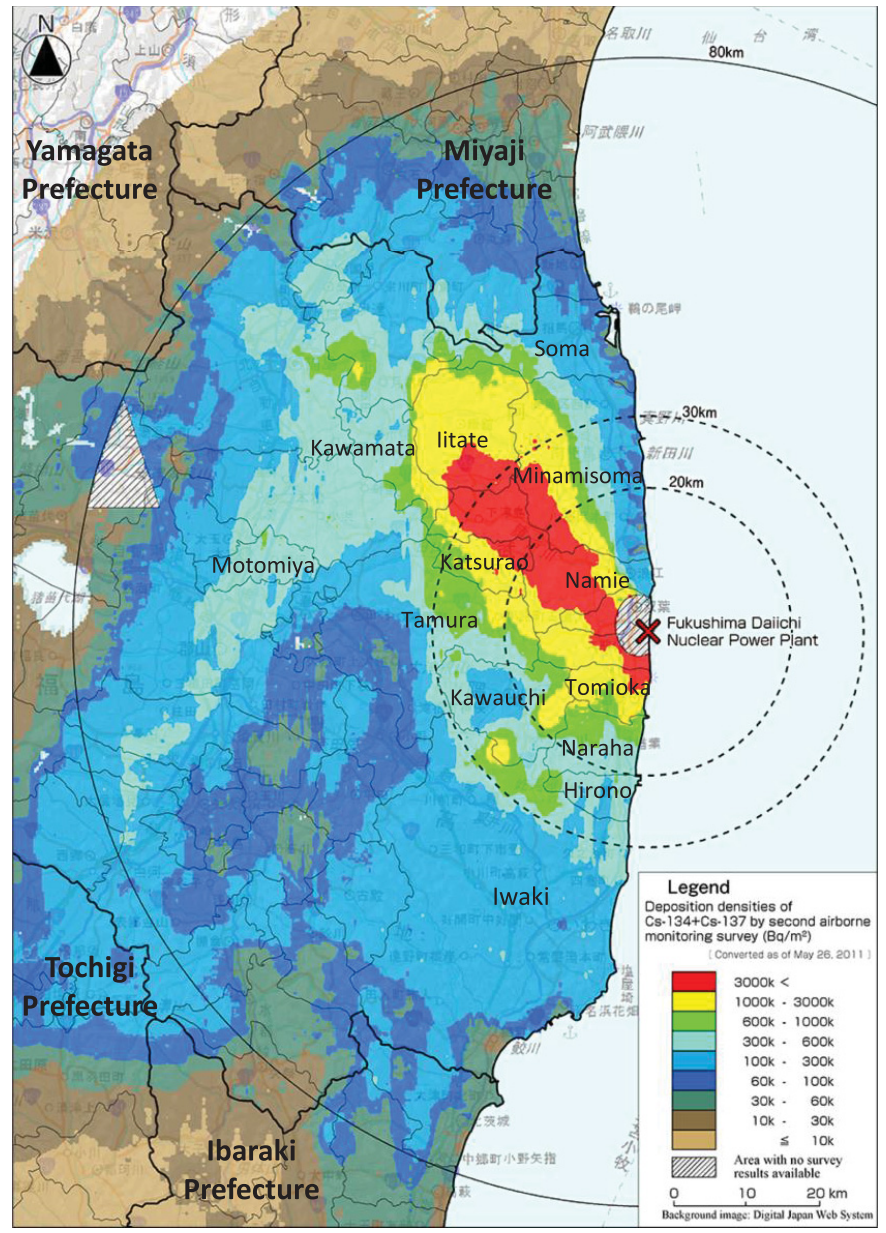

Fig. 1. Results of deposition of radio-caesium by airborne monitoring, converted as of May 2011 (Source: JAEA database for radioactive substance monitoring data).

winter), most crops had not yet developed, with the exception of a few market garden productions of leafy vegetables (e.g. spinach, salads, cabbage, kukitachina). Livestock was still mainly confined to barns and fed with fodder produced the previous year and often stored inside, protected from deposits (Renaud et al., 2013). Nevertheless, as illustrated in the Figure 1, significant levels of contamination were reached immediately after the first radioactive deposits in case of the most sensitive products (i.e. leafy vegetables), even in prefectures relatively far from the Fukushima NPP (e.g. Ibaraki, Toichigi, Gunma). Among the results published on the website of the MHLW as of 19 March 2011, one example is a sample of spinach leaves collected on 18 March 2011 in the city of Hitachi (120 km south of the NPP), with an iodine131 concentration of $54100 \mathrm{~Bq} / \mathrm{kg}$ fresh weight (MHLW, 2011a). On 21 March 2011, kukitachina leaves sampled in Motomiya (70 km west of the NPP) showed a concentration of radio-caesium of $82000 \mathrm{~Bq} / \mathrm{kg}$ fresh weight (Renaud et al., 2013). Fruit trees in early physiological stages (e.g. Japanese apricot trees, kiwi trees, Japanese persimmon trees, etc.) and evergreen plants (e.g. bamboo, tea trees, yuzu trees) were also affected by deposits, leading to leaves or fruit whose radiocaesium radioactivity reached several hundred $\mathrm{Bq} / \mathrm{kg}$ fresh weight during the second half of 2011. Similarly, the feeding of 
livestock using fodder stored outside at the time of the plume's passage led to iodine-131 contamination of meat and milk with the order of a few thousand $\mathrm{Bq} / \mathrm{kg}$ fresh weight (IRSN, 2016). For instance, iodine-131 contamination of $1190 \mathrm{~Bq} / \mathrm{kg}$ fresh weight was detected in milk produced in the city of Kawamata (Date District, Fukushima Prefecture) as of 16 March 2011.

In this context, the Japanese authorities undertook a series of measures to protect the population, starting with the emergency evacuation of the regions surrounding the Fukushima NPP. With regard to food safety management, rules concerning the food intended for human consumption were promulgated as of 17 March 2011, with the introduction by the MHLW of provisional standards for several artificial radionuclides, including iodine, caesium, uranium and plutonium (MHLW, 2011b). These provisional standards (equivalent to Maximum Permitted Levels, MPLs), expressed in $\mathrm{Bq} / \mathrm{kg}$ and adopted for five main food classes, were defined on the basis of the recommendations of international bodies such as the ICRP and the FAO-WHO Codex Alimentarius. It should be noted that if a standard is exceeded, this leads to the implementation of food restrictions (consumption or marketing restrictions) that can be lifted when the measurement results of the three consecutive weekly monitoring operations are found to be below threshold. As of 21 March 2011, following the first results obtained in regard to spinach leaves and fresh milk (see above), first marketing restrictions were issued in four prefectures (i.e. Fukushima, Ibaraki, Tochigi and Gunma). During the days that followed, new measurement results revealed contamination in other products, as well as in milk produced in other prefectures. Therefore, new food restrictions, in addition to the previous ones, were imposed on more products, both in Fukushima Prefecture (e.g. turnips, broccoli, parsley and other leafy plants, etc.) and in neighbouring prefectures (IAEA, 2015a). In the weeks and months that followed, the number of food restrictions continued to increase. Furthermore, as of April 2011, these restrictions were established specifically within each prefecture and even within each municipality. This led to the implementation of restrictions which differed quite significantly from one area to another. In addition, certain restrictions were sometimes tardy or non-exhaustive (Renaud et al., 2014). Consequently, a gradual loss of confidence of the Japanese population towards the authorities emerged (Renaud et al., 2014). Local products from the Fukushima Region began to suffer a loss of reputation, which intensified following the detection of radiological contamination in the Fukushima beef, a local emblematic product. Indeed, in June 2011, a single measurement with a concentration of $1800 \mathrm{~Bq} / \mathrm{kg}$ in the Fukushima beef, not only interrupted marketing in the entire meat sector (Steinhauser, 2017), but also led to a sudden loss of consumer confidence in all products from the Fukushima Prefecture. This was especially the case for Fukushima peach, other emblematic product whose sales had not yet been affected by the accident but abruptly fell by about 30\% (Takahashi et al., 2018).

Therefore, the occurrence of the Fukushima accident and the subsequent management strategies caused major disruptions in the agricultural sectors. It should be noted that in addition to progressive marketing restrictions, agricultural sectors were also significantly affected by the emergency evacuation orders, leading to the closure of many farms and the abandonment of nearly 40000 ha of agricultural land (MOE, 2018). However, at the end of 2011 , in order to define the conditions for the return of the population and to accelerate the resumption of activities, a post-accident zoning was defined, together with an ambitious programme of decontamination of living and agricultural areas. In parallel, various food management strategies were implemented within the different agricultural sectors. Then, several forms of collaboration between producers/livestock farmers, agricultural cooperatives, consumers and even distributors gradually emerged. These initiatives enabled the implementation of several actions, organised around three main goals:

- ensure the radiological quality of products by implementing countermeasures and monitoring systems;

- maintain sustainable economic activity by encouraging the resumption of agricultural activities and by deploying various sales strategies;

- restore consumer confidence by initiating producer/ consumer dialogues and promotional actions for local products.

The rest of this article aims to further detail the implementation of these three strategic pillars throughout the years following the Fukushima accident.

\section{Ensure the radiological quality of products}

\subsection{Implementation of agricultural countermeasures}

As of the end of 2011, the Japanese authorities undertook a decontamination programme of the regions affected by the Fukushima accident to subsequently foster the resumption of economic activities. In order to organise the work, the Japanese authorities established a zoning system classifying the affected regions into two main areas, based on their radiological level (see Fig. 2):

- The "Special Decontamination Area" (SDA), which is the area closest to the Fukushima Daiichi NPP and the most affected by the radiological contamination, corresponds approximately to the area evacuated following the accident (additional annual dose for individuals estimated to be over $20 \mathrm{mSv}$ in the first year after the accident). This area includes around 8500 hectares of agricultural land in eleven municipalities;

- The "Intensive Contamination Survey Area" (ICSA) corresponds to regions that were not evacuated but whose initial estimated ambient dose rate could lead to exposure greater than $1 \mathrm{mSv} /$ year. This area covers 92 municipalities (36 located in Fukushima Prefecture and 56 in neighbouring prefectures) and 32000 hectares of agricultural land.

Within the SDA, the Japanese government is responsible of developing and implementing the decontamination strategies. Concerning the ICSA, municipalities are conducting the remediation works, financially supported by TEPCO and technically supported by the national government who provided a set of guides detailing decontamination techniques (IAEA, 2015b; MOE, 2018). With regard to the decontamination 


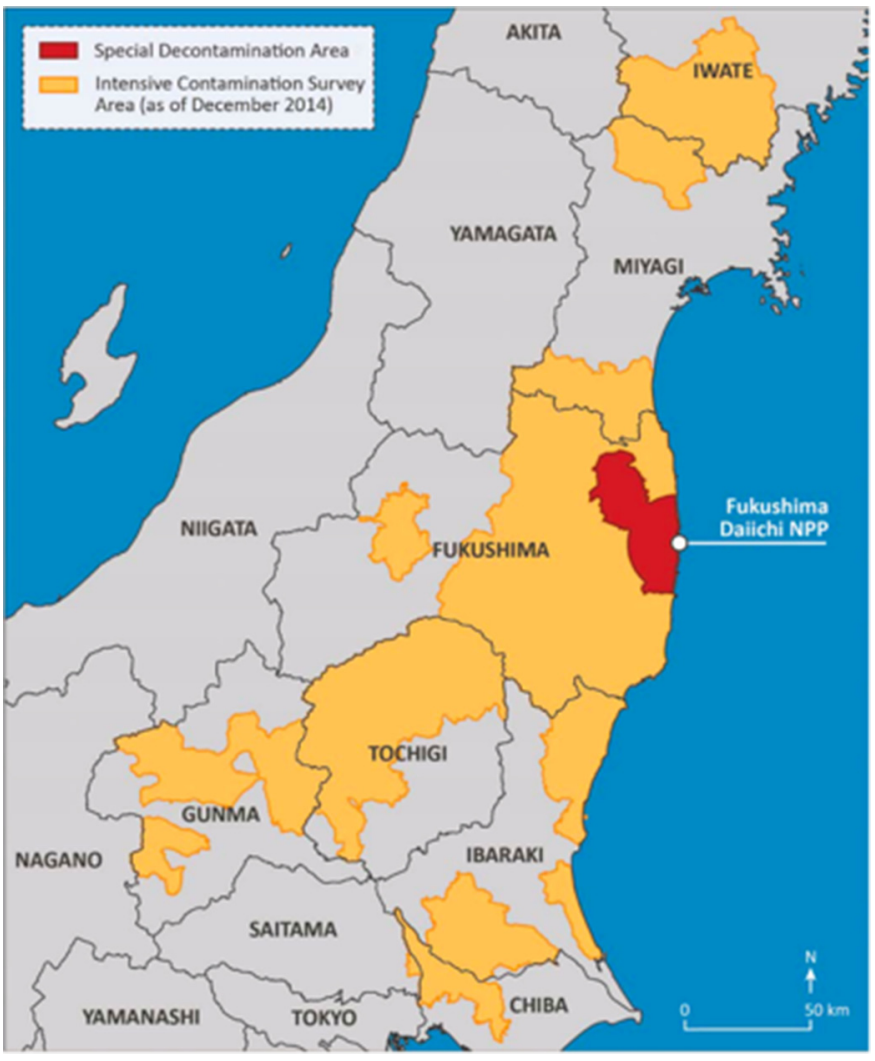

Fig. 2. The SDA and the ICSA (Source: IAEA, 2015b).

of agricultural areas, the MAFF (Japanese Ministry of Agriculture) published practical guides on the countermeasures that can be applied to agricultural land and production (MAFF, 2012).

The decontamination techniques applicable to both the ICSA and the SDA depend mainly on the level of contamination, as well as the type and nature of the soil. It should be noted that on the basis of existing data (e.g. feedbacks from the Chernobyl accident) and calculations of soil/plant transfer coefficients, the Japanese authorities considered that soil contamination of less than $5000 \mathrm{~Bq} / \mathrm{kg}$ of radio-caesium would lead to rice, fruit and vegetable production below the MPLs (Miyashita, 2012). Therefore, a soil concentration of less than $5000 \mathrm{~Bq} / \mathrm{kg}$ authorises the agricultural crops, whereas agricultural land with a soil concentration above $5000 \mathrm{~Bq} / \mathrm{kg}$ entails the implementation of countermeasures, using various techniques. As an illustration, the Table 1 summarises the various decontamination techniques applied in fields, pastures and orchards following the accident.

At the same time, specific countermeasures were carried out within farms to reduce the radiological contamination of milk or beef. Overall, these countermeasures consisted in (i) confining livestock to barns (measure still in effect in 2019 for some cases), (ii) providing a diet of "clean" fodder, grains or wild plants and, for dairy farming, fortifying fodder with magnesium and potassium to limit contamination transfers into milk, (iii) following a strict food monitoring process.
It should be noted that in the field, the practical works and the technical choices of countermeasures were often delegated to livestock breeder cooperatives, as well as to local agricultural cooperatives. For instance, Japan Agriculture Fukushima Miraï was highly involved in the organisation of decontamination work in its districts (e.g. Fukushima, Soma, Date, Adachi), and strongly encouraged its members to resume their agricultural activities thereafter. Consequently, in March 2018, the Japanese Ministry of Environment (MOE) announced that $100 \%$ of the decontamination of agricultural land had been completed within ICSA ( $c f$. MOE websitehttp://josen.env.jp/en/decontamination).

Several years after the Fukushima accident, while some measures have proved effective, feedback analysis of the implementation of agricultural countermeasures reveals significant consequences, on both environmental and human aspects. Although it contributed to reducing the ambient dose rate, decontamination operations and more specifically topsoil removal resulted in a considerable amount of waste. Currently, nearly 17 million cubic metres of waste have been produced and temporarily stored in more than a thousand locations.

In addition, it turns out that some agricultural countermeasures were sometimes ineffective. As an example, despite the drastic countermeasures used on Japanese persimmon trees (i.e. debarking, high-pressure water cleaning), the sales of anpokakis (i.e. variety of dried persimmon fruit, typical of the Date Region) could be authorized only in 2015, insofar as the contamination of the fruits was previously above the standards. The testimony of several livestock breeders also indicated that some countermeasures were counterproductive. This was particularly the case for the magnesium and potassium enrichment of fodder for dairy cows. This modified diet led to a mineral imbalance in the blood, which, according to some livestock farmers, may have led to the progressive poisoning of livestock, sometimes causing the premature death of dairy cows.

Finally, testimony of several farmers also reveal that the implementation of countermeasures generated a significant increase in work in terms of organisation, preventive measures, monitoring and additional costs due to the purchase of equipment and supplies. All these new concerns constituted stress factors that often led to a deterioration in farmers' living conditions, with serious psychological consequences, ranging from depression to suicide (Humbert-Amemiya, 2012).

\subsection{Radiological monitoring systems}

\subsubsection{New standards}

Alongside the introduction of agricultural countermeasures, reinforced radiological monitoring of food was organised as of March 2011 in the regions affected by the Fukushima accident and has continued to develop since then. As seen above, the implementation of this monitoring is based on a set of radiological criteria similar to the MPLs and referred to as "standards". These standards apply to both food products intended for export and those intended for the internal market.

In April 2012, after the analysis of the first results of the radiological monitoring carried out on the basis of the provisional standards published in 2011 by the MHLW (see part III), the Japanese authorities published national standards 
Table 1. Agricultural countermeasures implemented following the Fukushima accident (MAFF, 2014; IAEA, 2015b).

For fields and pastures

Agricultural countermeasures

Addition of potassium fertiliser

Reversal tillage

Topsoil removal $(5 \mathrm{~cm})$. Soil reconstitution based on

healthy soil and addition of potassium fertiliser

Weeding

Topsoil removal using solidification agents. Soil reconstitution

based on healthy soil and addition of potassium fertiliser

For orchards

High-pressure water jet cleaning or debarking of fruit trees

Pruning of small branches or leaf removal

Topsoil removal

Burial or incineration of the first harvest after the accident (in the event

of fruit contamination above the standards, in 2011: $500 \mathrm{~Bq} / \mathrm{kg}$ )
Action levels - Radio-caesium activity in soil (Bq/kg dry weight)

$<5000$

[5000-10 000]

[5000-25000]

$>5000$

$>5000$

Table 2. Values of standards defined for food in Japan (MHLW, 2012a).

\begin{tabular}{lll}
\hline & $\begin{array}{l}\text { Standards applied in 2011 } \\
\text { (for radio-caesium - Bq/kg) }\end{array}$ & $\begin{array}{l}\text { Standards applied in 2012 } \\
\text { (for radio-caesium - Bq/kg) }\end{array}$ \\
\hline Drinking water & 200 & 10 \\
Milk and dairy products & 200 & 50 \\
Food for children & - & 50 \\
Other food & 500 & 100 \\
\hline
\end{tabular}

for radio-caesium. This revision was based on a dosimetric reference level of $1 \mathrm{mSv} /$ year (instead of $5 \mathrm{mSv} /$ year) as well as new calculation assumptions (e.g. improved consideration of the radio-sensitivity of children, contribution of drinking water consumption to the internal dose through ingestion, etc.) (MHLW, 2012a).

Table 2 details the various standard values adopted by the Japanese authorities in 2011 and 2012 for radio-caesium.

It should be noted that today these standards are still much stricter than those recommended at the international level (e.g. Codex Alimentarius, European Regulation 2016/52/Euratom, etc.). This is due in particular to the consideration of a diet which is $50 \%$ based on potentially contaminated food, instead of the $10 \%$ used in most international regulations (Terada et al., 2018). However, it is worth noting that the change in standards announced in 2012, which reduced the provisional standards by a factor of 4 to 20, did not lead to an increase of food restrictions. Ultimately, this shows an overall improvement in the radiological situation (with regard to food) in just one year following the accident (IRSN, 2016).

In addition to national standards dedicated to food, standards were also introduced for animal feed. Currently, these concentration limits (in radio-caesium) are (Nakanishi and Tanoi, 2016):

$-100 \mathrm{~Bq} / \mathrm{kg}$ for equine and bovine feed;

$-80 \mathrm{~Bq} / \mathrm{kg}$ for pig feed;

$-160 \mathrm{~Bq} / \mathrm{kg}$ for poultry feed;

$-40 \mathrm{~Bq} / \mathrm{kg}$ for fish feed.

\subsubsection{Variety of measurement protocols drawing on several derived criteria}

To ensure compliance with standards, radiological monitoring systems were set up. For this purpose, ministries (e.g. MHLW, MAFF, MOE) published practical guides specific to production sectors, detailing the protocols, techniques and measurement frequencies to be observed. Prefectures and municipalities took charge of the practical implementation of radiological monitoring, in collaboration with farmers, livestock breeder federations and agricultural cooperatives. This monitoring generally consisted of carrying out weekly sampling and analysis of samples of fruits and vegetables or milk in dairy cooperatives' collection tanks. In slaughterhouses, systematic pre-marketing measurements (e.g. blood analysis, measurements on samples of neck and thigh meat) were also part of this institutional monitoring. For certain products such as the anpokaki (emblematic fruit of the Date Region hailed for its gustatory quality), or rice (a sensitive product in Japan, of which Fukushima Prefecture varieties are recognised for the quality of their taste), an exhaustive and automated system on test benches (screening) was developed to monitor $100 \%$ of production (Fig. 3).

Beyond these official measurements, Japanese feedback analysis shows that various stakeholders in the agricultural sectors took actions to ensure the radiological quality of their products. Indeed, following the methodological recommendations issued by the national authorities, the various agricultural sectors established their own radiological 


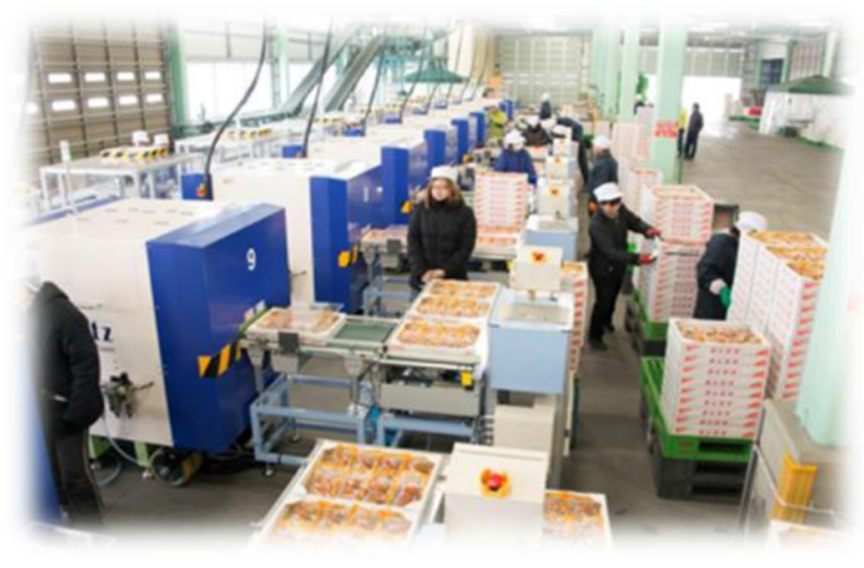

Fig. 3. Automated system on test benches developed for anpokakis (Source: Takahashi et al., 2018).

monitoring, often coordinated by agricultural cooperatives and livestock breeder federations. To this end, cooperatives equipped themselves to carry out measurements in parallel to the official monitoring system and to ensure monitoring on all production lines, in other words, "from the farm to the table". Beyond this, in order to achieve impeccable radiological quality, which is the primary requirement of consumers, derived radiological criteria (much stricter than the official standards) were introduced by the agricultural cooperatives themselves, at each stage of production. Discussions carried out with the various agricultural sectors show that these derived criteria were established independently by each of them, in order both to guarantee farmers the sale of their products and to provide consumers with food at contamination levels far below those of the standards. Three illustrations highlighting this strategy are provided below.

\subsubsection{Search for the origin of contamination}

To better identify the origin of the contamination and also to address consumer concerns regarding a possible dilution of contaminated milk in the course of collection, the Fukushima dairy cooperative decided to perform its own radiological monitoring. To this end, the dairy cooperative defined measurement points at each major stage of milk collection, each of which must comply with precise derived radiological criteria:

- at the level of each dairy farmer: criteria of $10 \mathrm{~Bq} / \mathrm{L}$ (in other words, five times less than the value of the official standard);

- at the collection truck level: criteria of $50 \mathrm{~Bq} / \mathrm{L}$ (value of the official standard set in 2012 for milk);

- at cooperative tank level: criteria of $50 \mathrm{~Bq} / \mathrm{L}$ (value of the official standard set in 2012 for milk).

\subsubsection{Anticipate any transfer phenomenon}

To prevent any transfer phenomenon through the ingestion of contaminated fodder, which could result in a high level of contamination of milk or meat, the dairy and agricultural cooperatives in Fukushima required their breeders to feed their livestock with fodder that does not exceed $30 \mathrm{~Bq} / \mathrm{kg}$ (and not $100 \mathrm{~Bq} / \mathrm{kg}$ as recommended by the standards set for animal feed).

\subsubsection{Adapt to measurement techniques and methods}

For the monitoring of anpokakis and rice, it was found that the screening technique used can lead to some artefacts that affect contamination measurement results ("false negatives"). To overcome this difficulty, derived radiological criteria to be respected during this monitoring were therefore defined (MHLW, 2012b):

- rice: $20 \mathrm{~Bq} / \mathrm{kg}$;

- anpokaki: $50 \mathrm{~Bq} / \mathrm{kg}$.

\subsubsection{Product traceability}

Radiological analyses carried out in the framework of the official monitoring and organised by the local authorities are traced and stored. For instance, the results of radiological analyses performed on Fukushima beef are recorded in a slip in which the reference number of the cattle is specified. Then, the meat is marketed with the following information clearly displayed: "measured", as well as the cattle identification number. The traceability of the product is thus ensured. The same traceability system is applied for anpokakis, for which a label is affixed to each tray put on sale. This confirms radiological monitoring and compliance with the applicable thresholds.

It should also be noted that Fukushima Prefecture regularly provides consumers with information on the principles and completeness of the monitoring operations carried out. Measurement results are available on the websites of the MAFF (www.maff.go.jp/e/policies/food_safety/index.html), the MHLW (www.mhlw.go.jp/english/topics/2011eq/index food.html) and THE Fukushima Prefecture (www.pref.fukush ima.lg.jp/site/portal-english/en01-01.html).

\subsubsection{Multiplicity of actors and radiological criteria}

The analysis of the radiological monitoring implemented in Japan over the past eight years clearly highlights a multiplication of forms of organisation in "self-checking" mode within each agricultural sector, in addition to official monitoring. The testimony received from the various agricultural sectors show that even today, the analyses performed on food are still carried out independently, with no attempt to pool resources between the members of the sectors and the local authorities. Similarly, no desire to share or compare results between these various actors are observed. The existence of multiple radiological criteria derived from official standards, which vary from one agricultural sector to another, also adds complexity to the monitoring system. Of note, these various monitoring strategies aim nowadays at radiological measurement results below the detection level.

\subsection{Specific case of sea fishing}

Sea fishing in the waters of the Fukushima Prefecture, whose two main ports are Soma and Iwaki, has been prohibited since 15 March 2011. Concerning the monitoring of sea fishing products, a specific strategy was developed by the national authorities in conjunction with the Fisheries Agency and placed under the responsibility of the Fukushima Prefecture. 
This strategy is reviewed and adapted based on the results of the radioactivity measurements performed on all edible marine products (e.g. fish, shellfish, cephalopods, etc.).

Since June 2012, fishing has been subject to a test phase ("trial fishing"): each time a boat returns to port, a sample of each species fished is taken for monitoring purposes. In the event of a "positive" measurement (that exceeds $50 \mathrm{~Bq} / \mathrm{kg}$ ), a prohibition on the fishing and marketing of the concerned species is imposed throughout the catch area and adjacent areas (inshore and offshore fishing areas are divided into sectors). Then, a programme of targeted measures concerning the species is launched. For at least three months, no samples from the twice-weekly sampling of the species shall exceed the standard, failing which the resumption of the fishing of the concerned species shall remain prohibited. In practice, since even slightly contaminated fish is rejected by consumers, it is only after several dozen confirmation measurements below an investigation threshold of $25 \mathrm{~Bq} / \mathrm{kg}$ that fishing for the species can effectively resume. The decision to resume fishing is taken jointly by a pluralist committee that brings together experts, scientists as well as representatives of the prefecture and local fishermen's associations. Since the implementation of this trial phase, sea trip times have been limited to one half a day per week instead of four days as before the accident, which de facto prevents the revitalisation of offshore fishing and reduces catch tonnage.

\section{Maintain sustainable socio-economic activity}

\subsection{Economic incentives for producers/livestock farmers}

After the Fukushima accident, the Japanese government sought to develop a system of indemnities and compensation for the entire population affected. The amounts allocated to the inhabitants of the affected areas depend on many parameters. Among these, the most important are the location of the property within the post-accident zoning defined by the authorities, the individual pre-accident situation of each person (e.g. wages or income from the previous two or three years, composition of the household) and the terms and duration of evacuation (e.g. self-evacuation or forced removal).

More specifically, as regards the socio-economic activities affected by the accident, the compensation system takes into account the drop in economic activity (in other words, drop in income, extraordinary costs linked to the interruption of activity, etc.), the loss of wages and the extra costs linked to radiological monitoring operations and waste management, as well as financial losses linked to marketing restrictions. For farmers, and in particular those who had to be evacuated, compensation also considers the loss of crops or livestock abandoned in March 2011. Upon farmers' return, after the lifting of evacuation orders, the costs related to the decontamination, cleaning and rehabilitation of living areas were also reimbursed. Extra-costs related to changes in production processes or techniques, the supply of specific raw materials (e.g. clean fodder), the implementation of radiological monitoring, or the drop in sales prices (in other words, loss of reputation) were also included in the reimbursements. However, in practice, testimonies from farmers, livestock breeder federations and agricultural cooperatives reveal that some of these expenses were compensated by TEPCO, but only for two or three years after the accident.

In parallel to the compensation system, local authorities (e.g. the prefecture and municipalities) set up several assistance and support mechanisms to boost the resumption of agricultural and industrial activities. For instance, in order to attract new entrepreneurs, local authorities notably offered them tax benefits. Logistical support for the rehabilitation or reconstruction of buildings and holdings was also provided by the authorities. However, despite all these efforts, the revitalisation of activity is turning out to be slow, particularly due to lack of local workers and to the loss of product reputation, which reduces sales.

Therefore, in the several years following the accident, economic and social activities in the affected regions were mainly supported through the compensation and indemnities system. On 1st April 2018, financial assistance to farmers to compensate for production and sales losses came to an end. However, financial assistance to sea fishermen will remain active until fishing has fully resumed. The government's decision to stop financial assistance to farmers is deemed unacceptable by professional associations, producer unions and associations of farming families. These associations considered that, nowadays, the countermeasures implemented and the associated radiological monitoring still lead to a significant increase in work. Farmers also have to bear additional costs, both in terms of equipment (e.g. measurement equipment) and in terms of fertilisers or "clean" feed for livestock. Moreover, farmers fear that drops in sales prices will still have to be borne for the several years to come. In this context, associations of farmers and livestock breeders regularly take legal action against TEPCO, in order to obtain an extension of compensation.

However, some actors also highlighted the ambivalent nature of this compensation system, which is often a source of inequity. For instance, farmers in regions located just outside evacuation areas receive very little assistance, despite the fact that their activities were also affected by radioactive deposits and they often face similar difficulties as their counterparts living inside the "post-accident zoning area" (e.g. a drop in prices, loss of reputation, quality control, etc.) (The Japan Times, 2018). The testimonies also revealed that some farmers decided not to resume their activity, since they considered it too stressful and no longer profitable. Furthermore, older farmers or fishermen close to retirement age have come to terms with the situation, preferring income from compensation rather than uncertain revenue from their disrupted activity. For their part, younger farmers and fishermen appear wanting to return to agriculture, livestock breeding or fishing under sustainable conditions.

\subsection{Product sales strategies faced with the loss of reputation}

In addition to the difficulties related to the implementation of countermeasures and radiological monitoring, the loss of reputation of products from the Fukushima Prefecture has also been a major obstacle to the recovery of the region's economic activities. Indeed, in the first few months after the accident, it 
often took only one sample measured above the "standard" to generate a crisis. This was the case with the contamination of the Fukushima beef in June 2011. Nowadays, similar situations are still observed, as for instance, each time a fish caught in the Pacific is found to be even slightly contaminated. As already raised by the European PREPARE research project (Charron et al., 2016), it must be stressed that an official standard that is exceeded is often interpreted by consumers as the crossing of a threshold involving a danger to health. This is because this system of "standards", based on technical aspects, is not always understood by consumers and can generate a feeling of insecurity. The result is a loss of confidence and an increase in fears regarding food safety that spreads to all products from the Fukushima Prefecture.

However, some typical and emblematic products of the Fukushima Region have suffered a relatively limited or at least less durable loss of reputation. Thus, it is observed that sales of anpokaki, traditional sake or other "flagship" products (e.g. the Rakuo-Café-au-Lait ${ }^{\circledR}$ from the Fukushima dairy cooperative) were ultimately relatively unaffected by the accident. It turns also that these products, whose excellence was recognised before March 2011, did not suffer from competition from other goods produced in prefectures not affected by the accident.

Therefore, it appears that the loss of reputation concerns products that can be easily substituted and that are or can easily be produced outside the affected areas, such as milk, rice, fruits and vegetables or fish.

Based on this observation, farmers, livestock breeder federations, agricultural cooperatives and distributors initiated new strategies for the sale of products from the regions affected by the Fukushima accident. The following paragraphs provide some illustrations of these strategies.

\subsubsection{The reduction of the sales price of local products}

To enable the resumption of the consumption of food produced in the Fukushima Prefecture, one of the levers frequently used by some distributors, and which de facto forces some producers to do the same, is to lower sales prices. Therefore, a price difference of 10 to $20 \%$ is still observed in 2019, particularly for rice, the Fukushima peaches or the Fukushima beef (MAFF, 2018). Discussions with members of the Fukushima Prefecture Chamber of Commerce indicate that although the origin of the product is important, it is mainly the price that has an influence on the buyer-consumer.

It should be noted that for some products, negotiations were undertaken with TEPCO to ensure that producers need not bear the drop in sales prices. In the case of milk for instance, prices were maintained through compensation acquired following negotiations initiated by cooperatives and professional associations with TEPCO and the government (milk price: $97 \mathrm{JPY} / \mathrm{L}$ before the accident; $98 \mathrm{JPY} / \mathrm{L}$ in March 2017).

Anpokakis also were not significantly affected by the fall in sales prices, for another reason. Indeed, after several years of production shortages (from 2011 to 2014), it turns that consumers did not hesitate to purchase these traditional dried fruits as soon as they were back on the market, at a price equivalent to that applied before the accident.

Discussions with consumer associations and producers also raised the ambiguous role sometimes played by distributors. Indeed, even though distributors buy the products from farmers/breeders at low prices throughout the year, putting forward the argument of the region's loss of reputation, it turns out that they adjust the sales price afterwards according to demand. For example, distributors tend to sell the Fukushima peaches at a reduced price all year round, except during the time of year when it is the only peach species available for sale in Japan.

\subsubsection{So-called "bypass" or reconversion strategies}

In addition to lowering the sales price of the Fukushima products, some sectors have also adopted "bypass" strategies, whose aim is to cancel out the negative reputation conferred on their products. For instance, the Fukushima agricultural cooperative, in collaboration with its distributors, decided to abandon the "Fukushima beef" label. Today, "Fukushima beef" meat is sold as "domestic beef" (without a designation of origin), enabling sales levels to be maintained (at a lower price), since buyers are no longer "warned" by the word "Fukushima".

An equivalent strategy was adopted by the Fukushima Dairy Cooperative. Since milk packs indicating their origin ("Fukushima milk") were stigmatised and "rejected" by consumers, unsold milk is now redirected to dairy cooperatives in neighbouring prefectures. These dairy cooperatives repackage Fukushima milk packs with the milk produced in their regions and market these new packs without mentioning the specific Fukushima origin. Therefore, it would appear that, for some products, the word "Fukushima" is sufficient to damage the reputation of a product and to dissuade consumers from buying it.

Reconversion is also a strategic option chosen by some producers in the Fukushima Prefecture. In this context, producers distinguished by their ability to develop a range of innovative processes. For instance, experiments were carried out by the Minkan inasaku kenkyujo association, created in 1999. Certified organic farming organisation in 2000, it aims to experiment with cultivation techniques and exchange experiences among its members within the framework of practices seeking to obtain stable yields, regardless of the climate and without the use of chemicals. Since March 2011, the Minkan inasaku kenkyujo association has been involved in maintaining agricultural activity in affected areas. On the least contaminated soils, the farmers who were members of this association experimented with the conversion of their rice fields into oilseed crop fields, in which better soil-plant transfer improves the absorption of caesium. This reconversion helped both to begin a process of soil decontamination and to maintain agricultural activity in the affected areas. Eleven farmers from Minamisōma participated in this project and planted 25 ha of plots with rapeseed, sunflowers and soybean (Ishii and Morlans, 2013).

\subsubsection{Overall improvement of product quality}

In an attempt to enhance the value of their products, farmers and breeders in the Fukushima Region worked not only to implement agricultural countermeasures, but also to improve the intrinsic and taste quality of their food produce. For example, beef producers in Fukushima decided to raise 
their animals using quality raw materials, right up to the selection of the best breeding bulls. Anpokaki producers, for their part, sought to produce fruit of a higher quality than that provided before the accident, in particular by growing larger fruit or seedless fruit. Similarly, methods of enhancing the value of seafood products are being implemented by various associations of fishermen. For instance, the possibility of the specialised fishing of exceptional products such as "Joban mono" anglerfish or turbot is being considered.

In the same spirit, organic farmer collectives of the Fukushima Region have particularly distinguished themselves in their ability to develop a range of innovative processes (e.g. above-ground cultivation, no pesticide use, no chemical fertiliser use, etc.), in order to improve the overall quality of their products (Orito, 2013).

\section{Restoring consumer confidence}

In addition to ensuring the radiological quality of products and attempting various commercial strategies, the interviews revealed that restoring consumer confidence has also become the shared ambition of producers, agricultural cooperatives, livestock breeder federations and some distributors (AugustinJean and Baumert, 2013). Alongside the many initiatives aimed at promoting the quality of local products, efforts have also been made to establish dialogue and build lasting links with consumers.

\subsection{Actions to promote local products}

Following the Fukushima accident, producers, distributors and some consumer cooperatives initiated numerous actions to promote products from the Fukushima Prefecture, thereby seeking to regain consumer confidence. For example, the "Coop. Fukushima", which is responsible for the distribution, marketing and promotion of local products and to which $35 \%$ of households in the Fukushima Prefecture adhere, has developed various initiatives to restore the reputation of products from the prefecture (NEA, 2016). Several information campaigns were organised via various channels: press articles, media reports, organisation of conferences, local products tasting sessions during local markets, fairs or traditional annual festivals, etc. Farmers and breeders themselves also sought to promote their products by offering visits to their farms and free tastings. The Fukushima Chamber of Commerce also plays a very active role in promoting the products of the entire Fukushima Prefecture to the local population and to population living outside the prefecture. It should be noted that several "media events" were also attempted to enhance the value of the Fukushima products among the Japanese and international population. For instance, the prime minister and the Emperor of Japan posed while tasting the Fukushima rice and peaches. To show its solidarity, the Yoshinoya ${ }^{\circledR}$ fast-food chain communicated on its choice to buy products from the Fukushima Prefecture for its restaurants.

Promotional actions for products from the Fukushima Prefecture are also carried out with respect to "external" consumers. For example, stands dedicated to seafood products from Fukushima are set up in various stores in Tokyo. Awareness-raising activities are also provided for young schoolchildren and national and foreign students to show them the reality of the situation in the regions of the Fukushima Prefecture. During the visits organised in these regions, meetings with livestock farmers and producers are proposed, as well as measurements in local radiological measurement centres. The goal of these visits is to promote a positive image of the region and its specialities.

\subsection{Innovative partnerships-producer/consumer link}

Since the early 1960s, a tradition of partnerships between agricultural producers and consumers has been maintained in Japan (Lagane, 2013). These partnerships or teikei are based on a direct "person-to-person" relationship (e.g. direct sales, short marketing channels) that promotes mutual recognition and confidence. They are established on the basis of an ethical charter and are intended to promote "local, civic, healthy and supportive small-scale agriculture" (Orito, 2013). In the 1990s, this form of agricultural partnership declined, with barely a thousand teikei systems still identified. After the Fukushima accident and in a climate of consumer mistrust towards the authorities, this type of partnership found renewed interest. The survival of these teikei, based on the consideration of local knowledge, also encouraged the deployment of mutual assistance and solidarity practices and sometimes strengthened the cohesion of affected communities.

In the same spirit, local associations and sometimes even the families of producers organised themselves to prepare baskets or lunch boxes (i.e. bento) using exclusively local products. These meal baskets contain products of undeniable gustatory and radiological quality (i.e. contamination levels below official standards) and are intended for local sale.

Another example of collaboration between consumers, producers and distributors is the Nouminren farmers' federation (www.nouminren.ne.jp). Created in 1989, this federation of farmers and breeders now has nearly 50000 members throughout Japan, who adhere to specific agricultural practices (e.g. family farming, restricted use of pesticides, absence of GMOs, etc.). This federation has its own test laboratories, capable of measuring in food products the presence of insecticides, heavy metals and other chemicals and now additionally the presence of radioactivity. Analyses are carried out in the laboratory and then discussed at the level of each prefectoral branch. Indeed, dialogue units that bring together consumer and distributors' representatives (including cooperatives) are set up to discuss expectations for the quality of the products sold by the Nouminren network. During these discussions, maximum levels of all types of contaminants, sampling protocols and their frequencies are defined through mutual agreement. Then, Nouminren prefectoral branches ensure that their farmers and livestock breeders comply properly with the agreed protocols and their shops market these "clean" products for direct sale. In this context, following the Fukushima accident, discussions among producers, consumers and distributors in the Fukushima branch led to agreement on the fact that radiological criterion had to be added to the other criteria used to determine the overall quality of products from the Fukushima Prefecture. This genuine triangular relationship of confidence between producers, distributors and consumers, established long before the Fukushima accident, was thus able 
to limit the impacts of the accident on the products marketed (in particular milk and dairy products) by the farms of the Nouminren Federation.

\subsection{Feedback on consumer reactions}

Despite all these promotion and dialogue efforts, producers, distributors and cooperatives observe that several years after the accident, some of consumers react in very different ways. For example, within the Fukushima Prefecture itself:

- some consumers remain anxious and concerned about radiological contamination. They have changed their eating habits by boycotting all products from the Fukushima Prefecture, since their origin is sufficient to make them suspect (from these consumers' point of view). Thus, these consumers tend to buy products from neighbouring prefectures, considering them safer, while paradoxically, no analysis attests to their radiological quality (Koyama, 2013);

- other consumers are ultimately convinced by the actions to promote local products, and dialogue initiatives mentioned above and appear to have faith in the monitoring system implemented by the authorities.

Regarding consumers living outside the Fukushima Prefecture, it is seen that following the accident, some consumers bought products from the Fukushima Region in a spirit of solidarity, but without much conviction as to their radiological quality. Furthermore, it is likely that some of these products were not consumed. Solidarity purchasing as a "social value" contributing to the restoration of the affected areas was therefore a motivation for external consumers in the first months following the accident (Suzuki et al., 2019).

However, the analysis of consumer behaviour shows some evolutions over the years. Consumers living in the regions affected by the accident were anxious about the radiological quality of products in the first few months after the accident. Through the dialogue with producers, cooperatives and federations of livestock farmers, these fears gradually dissipated. Beyond compliance with official standards (whose numerical values are of little importance to them), these consumers nonetheless pay careful attention to the fact that analytical results demonstrate the absence of contamination $(0 \mathrm{~Bq})$ or at least that values are below the detection limit ("undetected"). Thus, no longer fearing the presence of radioactivity in food, these consumers give priority to the gustatory quality of the products provided to them, as well as their prices. A survey conducted in February 2019 among consumers living in the regions affected by the accident (CAA, 2019) shows that $29.7 \%$ of their purchases are based on the product's gustatory quality, $22.1 \%$ on the product's price, $20.8 \%$ on the product's freshness and only $15.6 \%$ on the absence of radionuclides in the product. For their part, external consumers are also gradually regaining confidence in the Fukushima products. This is particularly being fostered through dialogue with consumers established in these regions (Suzuki et al., 2019). Therefore, Japanese feedback (Ban, 2016) shows the importance of establishing lasting dialogue with consumers, both on issues related to the radiological aspects and on those concerning the intrinsic quality of the food produce. Already observed during the work of the European PREPARE research project (Charron et al., 2016; Raskob, 2016), this is a major lever for restoring consumer confidence.

\section{Conclusion}

The analysis of feedback on the management of contaminated goods in Japan following the Fukushima accident shows that many strategies are set up to regain consumer confidence. These strategies involve multiple actors (e.g. producers, livestock farmers, distributors, cooperatives, local and national authorities, consumers' associations) who are all committed, each in their own way and often in collaboration, to ensuring the quality of the local products as well as their promotion. The "standards" established by the Japanese authorities following the accident enabled the definition of rules concerning the safety of food intended for human consumption. However, feedback reveals that in order to assess the radiological quality of the products they buy, local and even national consumers are much more likely to refer to the concepts of " $0 \mathrm{~Bq}$ added" or "undetected radioactivity". This consumer attitude led the various agricultural sectors to make considerable efforts in terms of radiological monitoring (establishing numerous derived radiological criteria) and countermeasures.

In parallel, exceptional measures were implemented to revitalise economic activity in the Fukushima Region, where profound social and demographic changes brought about by the accident (and the prolonged evacuation orders) are now changing its future prospects. Although a gradual desertification of the rural areas of the Fukushima Prefecture and a lack of interest on the part of younger generations to work in the agricultural sector was observed before the disaster, the accident in March 2011 greatly accelerated the impoverishment of the rural fabric. Today, we can see that the affected areas are undergoing major changes: several innovative projects (e.g. photovoltaic farms, waste treatment technologies, robotics, drone engineering, etc.) supported by the government are emerging as part of an economic pivot. In this type of context, questions regarding the sustainable resumption of agricultural activities in this region may arise. Even though all evacuation orders have been lifted, with the exception of a few areas still classified as "difficult to return to", very few young farmers and livestock breeders have returned to live and work on agricultural land. Moreover, these young farmers and livestock farmers are now facing economic difficulties $(e . g$. strong competition from producers in other regions, increased production costs, lower prices, cessation of compensation since 1st April 2018) as well as difficulties in mobilising workers, who have become rare. In the field of sea fishing, there are difficulties in recovering activity since to date (2019), the only authorised fishing expeditions are carried out as part of a trial phase that limits both fishing times and areas, as well as catches. Therefore, all these elements are obstacles to the revitalisation of stable, profitable and sustainable food production business.

Moreover, the future of the radiological monitoring system developed to check the quality of goods may come into 
questions. Indeed, the fact that nowadays, almost all the results of radioactivity measurement in food products show concentrations below detection limits pleads for a re-adaptation of the monitoring system, while maintaining long-term vigilance.

Finally, regarding the radiological quality of local products, it appears that confidence among consumers living in the Fukushima Prefecture has greatly improved, but this is less true for consumers living outside the Prefecture. In the future, it would therefore be wise to further explore the changes in these consumers' behaviour, to consider how to support and promote the effectiveness of the partnerships between producers and consumers that are already in place within the Fukushima Prefecture, and to highlight distributors' role and opportunities in ensuring the agro-economic resilience of the affected regions.

\section{Acknowledgments}

Analyses partly funded by the PREPARE European research project (as part of the OPERRA Project) and the CONFIDENCE European research project (as part of the CONCERT project) have been considered for this paper. PREPARE project has received funding from the European Atomic Energy Community Seventh Framework Programme FP7/2012-2013 under grant agreement 323287. CONFIDENCE project has received funding from the Euratom research and training programme 2014-2018 under grant agreement No.662287. This publication reflects only the author's view. Responsibility for the information and views expressed therein lies entirely with the authors. The European Commission is not responsible for any use that may be made of the information it contains.

\section{References}

Augustin-Jean L, Baumert N. 2013. Les réactions des consommateurs japonais suite à la contamination nucléaire de mars 2011 et leurs conséquences sur le rapport au territoire. Géographie et cultures 86: 49-64.

Ban N. 2016. Japanese experience in stakeholder involvement: ICRP dialogue meetings. Radioprotection 51(HS1): S51-S53.

CAA. 2019. 12th Fact-finding survey of consumers' awareness of reputation damage. Available from https://www.caa.go.jp/disas ter/earthquake/understanding_food_and_radiation/pdf/understan ding food and radiation 190306 0003.pdf (in Japanese).

Charron S, Lafage S, Van Asselt E, Baptista M, Van Bourgondie NM, Brandhoff P, Cabianca T, Camps J, Cessac B, Crouail P, Durand V, Gallego E, Gil O, Holmes S, Hourdakis C, Jones K, Kamenopoulou V, Lecomte J-F, Liland A, Lopes I, Madruga M-J, Martins J-O, Mc Mahon C, Montero M, Murith C, Olyslaegers G, Organo C, Paiva I, Peltonen T, Portugal L, Potiriadis C, Prades A, Reis M, Rossignol N, Schneider T, Sala R, Smith V, Tafili V, Teles P, Tomkiv Y, Trueba C, Turcanu C, Turtiainen T, Twenhöfel C, Vaz P. 2016. Overview of the PREPARE WP3: Management of contaminated goods in postaccidental situation-Synthesis of European stakeholders' panels. Radioprotection 51(HS2): S83-S91.

Humbert-Amemiya H. 2012. Fermiers sinistrés de Fukushima: comment faire face dans des conditions extrêmes? Ebisu 47: $165-172$.
IAEA. 2015a. The Fukushima Daiichi accident. Technical Volume 3/5, Emergency preparedness and response.

IAEA. 2015b. The Fukushima Daiichi accident. Technical Volume 5/5, Post-accident recover.

IRSN. 2016. Suites de l'accident nucléaire de Fukushima en mars 2011. Contamination des denrées japonaises. Point de situation en mars 2016.

Ishii K, Morlans S. 2013. La reprise des activités agricoles dans les régions contaminées après l'accident de Fukushima : un défi lancé aux agriculteurs. Géographie et cultures 86: 65-82.

Kotoba. 2015. Dialogues in Fukushima. The story of four years of dialogue for the rehabilitation of living conditions in the areas contaminated by the Fukushima accident, Web documentary. Available from http://www.fukushima-dialogues.com.

Koyama R. 2013. Damage to agricultural land and a radioactive substance inspection system in Fukushima prefecture. In: Workshop NERIS-ConGoo Madrid.

Lagane J. 2013. L'apport des partenariats solidaires entre producteurs agricoles et consommateurs en temps de crise. Géographie et cultures 86: 101-117.

Lochard J, Schneider T, Ando R, Niwa O, Clement C, Lecomte JF, Tada JI. 2019. An overview of the dialogue meetings initiated by ICRP in Japan after the Fukushima accident. Radioprotection 54 (2): 87-101.

MAFF. 2012. Technical guidelines on farmland decontamination: Monitoring, planning and implementation (in Japanese). Available from http://www.maff.go.jp/j/press/nousin/noukan/pdf/ 120831-08.pdf.

MAFF. 2014. Efforts for the reconstruction assistance of agriculture, forestry and fisheries after the Great East Japan earthquake (in Japanese). Available from http://www.maff.go.jp/j/kanbo/ kihyo02/fukkou/pdf/2612 zen.pdf.

MAFF. 2018. Report on fact-finding survey of distribution of agricultural products from Fukushima 2018 (in Japanese). Available from https://www.maff.go.jp/j/shokusan/ryutu/attach/ pdf/190329-10.pdf.

MHLW. 2011a. Levels of radioactive contaminants in foods tested in respective prefectures. sum up of radionuclide test results of food sampled since 19 March 2011 until 31 March 2012. Available from https://www.mhlw.go.jp/english/topics/2011eq/index_food radioactive.html.

MHLW. 2011b. Specifications and standards for foods, food additives, etc. Under the Food Sanitation Act.

MHLW. 2012a. New standard limits for radionuclides in foods. Available from http://www.mhlw.go.jp/english/topics/2011eq/dl/ new_standard.pdf.

MHLW. 2012b. Screening method for radioactive cesium in food products.

Miyashita K. 2012. Minimizing the contamination of agricultural environment toward food safety-With primary focus on the Fukushima nuclear disaster in 1998-2018. Food and Fertilizer Technology Center Database.

MOE. 2018. Environmental remediation in Japan. Available from josen.env.go.jp/en/pdf/progressseet_progress_on_cleanup_ef forts.pdf.

Nakanishi TM, Tanoi K. 2016. Agricultural Implication of the Fukushima nuclear accident - The first three years. Springer Open publications.

NEA. 2016. International Workshop on Post-Accident Food Safety Science, Summary Report, JT03428824.

Ohara T, Miyahara K. 2016. An overview of progress in environmental research on radioactive materials derived from the Fukushima nuclear accident. Glob. Environ. Res. 20: 001-014. 
Orito E. 2013. Les Teikei - les précurseurs au Japon de l'agriculture biologique - face à la catastrophe nucléaire de mars 2011 . Géographie et cultures 86: 83-99.

Raskob W. 2016 The PREPARE project - Innovative integrated tools and platforms for radiological emergency preparedness and postaccident response in Europe. Radioprotection 51(HS1): S55-S57.

Renaud P, Simon-Cornu M, Gonze M-A, Mourlon C, Parache V, Korsakissok I, Navarro E, Cessac B, Rannou A, Champion D. 2013. Contamination of Japanese foodstuffs of terrestrial origin after the Fukushima nuclear accident and related dose assessments. Part 1: Foodstuff Contamination. Radioprotection 48(4): 493-509.

Renaud P, Simon-Cornu M, Gonze M-A, Mourlon C, Parache V, Korsakissok I, Navarro E, Cessac B, Rannou A, Champion D. 2014 Contamination of Japanese foodstuffs of terrestrial origin after the Fukushima nuclear accident and related dose assessment. Radioprotection 49(1): 17-22.

Steinhauser G. 2017. Monitoring and radioecological characteristics of radio-cesium in Japanese beef after the Fukushima nuclear accident. J. Radioanal. Nucl. Chem. 311(2): 13671373.

Suzuki T, Oishi T, Kurokura H, Yagi N. 2019. Which aspects of food value promote consumer purchase intent after a disaster? A case study of salmon products in disaster-affected areas of the Great East Japan earthquake. Foods 8(1): 14

Takahashi H, Sato K, Takata D, Hayashi K. 2018. Damages of fresh and processed fruit industries imposed by the 2011 Fukushima Dai-ichi nuclear accident and counteractions implemented by the local agricultural co-operative - The case of Peach and Ampogaki (Semi-dried Persimmon) in Date City. Available from https:// territories.eu/assets/files/dissemination-presentations/bordeaux2018/2_Takahashi_Sato_Takata_Hayashi_English.pdf.

Terada H, Yamaguchi I, Shimura T, Svendsen E-R, Kunugita N. 2018. Regulation values and current situation of radioactive materials in food. J. Natl. Inst. Public Health 67(1): 21-33.

The Japan Times. 2018. Tepco's compensation for 3/11 victims has made matters worse for many (Published on13 Avril 2018).

Cite this article as: Maître M, Croüail P, Durand V, Lecomte J-F, Charron S, Schneider T. 2020. The management of contaminated goods in Japan since the Fukushima accident. Radioprotection 55(1): 17-28 